\title{
Auger Effect Revisited: An Essay by Inelastic Collision Theory
}

\author{
Alexandre M. Dias \\ Universidade José do Rosário Vellano, Alfenas, Brazil \\ Correspondence to: Alexandre M. Dias, alexandre.dias@unifenas.br \\ Keywords: Auger Effect, Collision Theory
}

Received: December 18, $2017 \quad$ Accepted: February 11, 2018

Published: February 14, 2018

Copyright $\odot 2018$ by authors and Scientific Research Publishing Inc.

This work is licensed under the Creative Commons Attribution International License (CC BY 4.0).

http://creativecommons.org/licenses/by/4.0/

(c) (i) Open Access

\section{ABSTRACT}

This work presents an essay to the Auger Effect, by the Inelastic Collision Theory. Calculations of the energies of the electrons ejected for bands of the Auger spectrum of some molecules were made, to test of the model.

\section{INTRODUCTION}

Auger Effect [1] observed with atoms consists of a nonradioactive electron transition, with ejection of an electron from an initial state of the atom with a "hole" in the innermost electron layer.

A neutral atom receives initially, a radiation of energy E, capable of withdrawing an electron from its electron layer K, leaving it with a "hole" in this layer. For light atoms, there is a great possibility that such a hole is filled by an electron from the outer electron layers, by a nonradioactive transition. This transition is accompanied by the ejection of an electron with kinetic energy T. The spectrum of these ejections is obtained experimentally by measuring the relative intensity of the electrons ejected at various values of $\mathrm{T}$.

Figure 1 illustrates the normal process, showing one of the possible final states of the system. In this

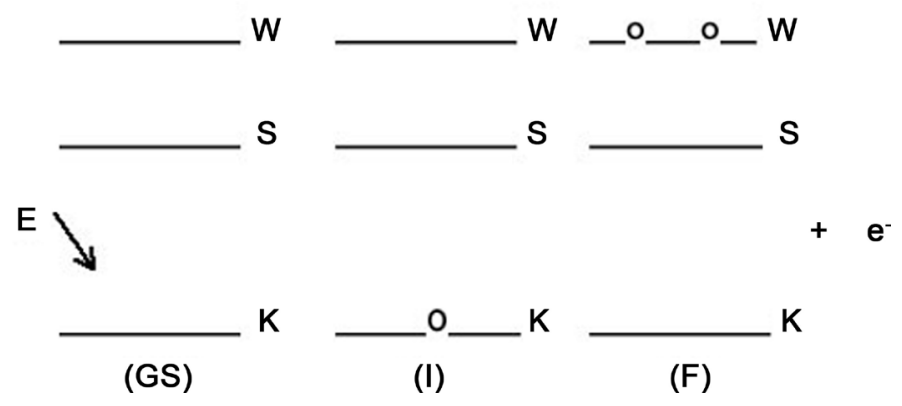

Figure 1. Effect Auger Normal K-WW. The small circles (O) represent "holes" in the electronic layer (I) and (F) represent the initial and final states considered in the process. (GS) stands for the "Ground State" system. 
figure, W represents "weakly bonded" valence electrons and S represents "strongly bonded" valence electrons $[2,3]$.

The normal Auger process corresponds to the transitions represented by K-WW, K-WS and K-SS. The first letter represents the layer with the "hole" in the initial state (I) and the next two letters represent the layers with "holes" in the final state (F) of the system. The transitions corresponding to these normal Auger processes lead to more intense lines in the Auger spectrum. Several other "satellite lines" in the Auger spectrum are identified by transitions involving an initial excitation of a K-layer electron into a discrete state.

\section{INTEREST BY THE AUGER EFFECT}

As the transition energy depends, at first approximation, on the binding energy of the electron $\mathrm{K}$, the Auger spectrum constitutes, in a sense, an "X-ray" of the system. Thus, this process has been used as an efficient impurity detection technique on material surfaces.

Industrial processes involving the machining of precision and impurities free surfaces, such as the manufacture of disks for microcomputers, may employ the Auger process for the determination of probable unwanted impurities on these surfaces. This is an example of the importance of the domain of this technology and of the interest of the molecular physicists by the study of this process all over the world [4-8].

\section{THEORY}

The shock section for the ionization of a hydrogen atom in the Ground State (GS), with ejection of an electron with energy $x^{2} / 2$ in the direction $\vec{x}$, was obtained by Massey and Mohr in 1933 [9], as:

$$
\mathrm{d} \sigma_{x}=\frac{8 \pi}{k^{2}} \frac{\mathrm{d} x}{x^{3}}
$$

$\mathrm{d} \sigma_{x}$ is equivalent to the Rutherford formula [8], for the shock section relative to the energy interval $\mathrm{d} \varepsilon$, white conditions for impulsion $q \gg\left(1 / a_{0}\right)$, where $a_{0}$ is of the order of dimensions atomic:

$$
\mathrm{d} \sigma_{\varepsilon}=\frac{\pi e^{4}}{E} \frac{\mathrm{d} \varepsilon}{\varepsilon^{2}}
$$

Here, $E$ is the energy of the incident electron and $\varepsilon$, the energy of the scattered electron, which, by analogy with Equation (1), corresponds to the energy of the ejected electron.

The shock section related to energy transfer $\left(E-E_{0}\right)$ (system energy difference after and before collision) is related to the loss energy of the incident electron through Differential Effective Braking [9], given by:

$$
\mathrm{d} E=\sum_{n}\left(E_{n}-E_{0}\right) \mathrm{d} \sigma_{3}
$$

Here, both can be over the states of the discrete spectrum as over the continuous spectrum, and $\mathrm{d} E$ represents the average energy lost per electron within a given solid angle.

It is "reasonable" to assume that the loss energy is proportional to the variation energy of the ejected electron [2]. Let us then admit the relation:

$$
-\frac{\mathrm{d} E}{\mathrm{~d} \varepsilon}=c
$$

It is reasonable to assume that the loss of energy is proportional to the energy variation of the ejected electron. Let us then admit the relation: $\mathrm{d} E \cong E_{k} \mathrm{~d} \sigma_{n}$, where $E_{k}=E_{0}-E_{n}$, where $E_{k}$ is the energy required to ionize the electron $K$, which corresponds to the first phase of the Auger process. From Equation

(2) $\mathrm{d} \varepsilon \cong \varepsilon^{2} \mathrm{~d} \sigma_{\varepsilon}$. Therefore, we have $-\mathrm{d} E / \mathrm{d} \varepsilon \cong E_{k} / \varepsilon^{2}$.

From experience [2], we can observed that the relation between energy variation to the ionize electron $K$ 
and energy variation to the ejection electron, is approximately constant:

$$
\frac{\left|\Delta E_{k}\right|}{\left|\Delta \varepsilon_{B}\right|^{2}}=\frac{\left|E_{k}\left(\mathrm{~N}_{2}\right)-E_{k}\left(\mathrm{O}_{2}\right)\right|}{\left|\bar{\varepsilon}_{B}\left(\mathrm{~N}_{2}\right)-\bar{\varepsilon}_{B}\left(\mathrm{O}_{2}\right)\right|^{2}} \cong \frac{\mid E_{k}\left(\mathrm{~N}_{2}\right)-E_{k}(\mathrm{O} \text { de CO }) \mid}{\mid \bar{\varepsilon}_{B}\left(\mathrm{~N}_{2}\right)-\left.\bar{\varepsilon}_{B}(\mathrm{O} \text { de CO })\right|^{2}} \cong 0.007
$$

So, we are going to infer that $c \cong 0.9 E_{k}^{3} / \varepsilon^{6}$, and:

$$
\mathrm{d} E \cong 0.9 \frac{E_{k}^{3}}{\varepsilon^{6}} \mathrm{~d} \varepsilon
$$

Now, from Equations ((3), (4) and (6)), one can obtain that

$$
0.9 E_{k}^{3} \frac{\mathrm{d} \varepsilon}{\varepsilon^{6}} \cong\left(E_{G S}-E_{f}\right) \mathrm{d} \sigma_{\varepsilon}
$$

where, $E_{G S}=E_{0}$ represents the energy of the system's ground state, before ionization, and $E_{f}=E_{n}$, the final energy of the system.

From Equations ((2) and (7)), we obtain

$$
\mathrm{d} \varepsilon \cong \frac{\varepsilon^{6}}{0.9 E_{k}^{3}}\left(E_{G S}-E_{f}\right) \mathrm{d} \sigma_{\varepsilon}
$$

With this result, remembering that $k^{2}=\left(2 m E / \hbar^{2}\right)$, using Equation (2) and the atomic units $m=1$, $e=1, \hbar=1$ and the condition $E=E_{k}$ required for that Auger Effect occurs, we finally have,

\begin{tabular}{|c|c|c|c|c|c|c|}
\hline Molecule & Auger Effect & Spectral line & $E_{k}$ & $E_{G S}-E_{f}$ & $\varepsilon_{f G S}$ & $\varepsilon_{\exp }$ \\
\hline & $\mathrm{GS} \rightarrow \mathrm{K}-\mathrm{WW}$ & B & 409.9 & 34.2 & 363.1 & 358.7 \\
\hline \multirow[t]{3}{*}{$\mathrm{N}_{2}$} & K-WS & $\mathrm{C}$ & & 55.4 & 309.2 & 337.9 \\
\hline & K-SS & $\mathrm{D}$ & & 76.6 & 277.5 & 315.0 \\
\hline & $\mathrm{GS} \rightarrow \mathrm{K}-\mathrm{WW}$ & B & 544.2 & 37.6 & 513.4 & 496.2 \\
\hline \multirow[t]{2}{*}{$\mathrm{O}_{2}$} & K-WS & $\mathrm{C}$ & & 59.4 & 440.7 & 466.4 \\
\hline & K-SS & $\mathrm{D}$ & & 81.2 & 397.2 & - \\
\hline \multicolumn{7}{|l|}{$\mathrm{CO}$} \\
\hline \multirow{3}{*}{$\mathrm{C}$} & $\mathrm{GS} \rightarrow \mathrm{K}-\mathrm{WW}$ & B & 295.9 & 34.6 & 234.2 & 248.3 \\
\hline & K-WS & $\mathrm{C}$ & & 55.6 & 200.0 & 225.6 \\
\hline & K-SS & $\mathrm{D}$ & & 76.6 & 179.7 & - \\
\hline \multirow{3}{*}{$\mathrm{O}$} & $\mathrm{GS} \rightarrow \mathrm{K}-\mathrm{WW}$ & $\mathrm{B}$ & 542.1 & 34.6 & 525.1 & 492.3 \\
\hline & K-WS & $\mathrm{C}$ & & 55.6 & 448.3 & 464.3 \\
\hline & K-SS & $\mathrm{D}$ & & 76.6 & 402.8 & 442.3 \\
\hline
\end{tabular}

Table 1. Summary of results. All energies are in $\mathrm{eV}$. The column $\varepsilon_{f G S}$ represents the average energy calculated by Equation (9) of this work. The column $\varepsilon_{\text {exp }}$ represents the average experimental energy obtained from the energies measured for each band by Moddeman et al. [2]. 


$$
\varepsilon_{f G S} \cong \frac{0.9 E_{k}}{\left[\frac{4 \pi}{E_{k}}\left(E_{G S}-E_{f}\right)\right]^{\frac{1}{3}}}
$$

where, $\varepsilon_{j G S}$ provides the average energy of the electrons ejected for each $G S \rightarrow F$ process, accompanied by the Auger Effect.

The results obtained by Equation (9) were compared with the mean energies for each band B, C, and $\mathrm{D}$ of the spectrum of molecules $\mathrm{N}_{2}, \mathrm{O}_{2}$ and $\mathrm{CO}$, obtained experimentally by MODDEMAN et all [2].

In order to obtain $\left(E_{G S}-E_{f}\right)$, the same scheme of the work of MODDEMAN et all [2], was used at light of Koopman's theorem, that is

$$
\left(E_{G S}-E_{f}\right)= \begin{cases}2 \varepsilon_{1}, & \text { para o processo } \mathrm{GS} \rightarrow \mathrm{K}-\mathrm{WW} ; \\ \varepsilon_{1}+\varepsilon_{2}, & \text { para o processo } \mathrm{GS} \rightarrow \mathrm{K}-\mathrm{WS} ; \\ 2 \varepsilon_{2}, & \text { para o processo GS } \rightarrow \mathrm{K}-\mathrm{SS} .\end{cases}
$$

Table 1 summarizes the comparison of results.

\section{CONCLUSION}

As can be seen in Table 1, the mean energies calculated by Equation (9) of this work represent a reasonable approximation for the experimental average energies. That experimental results for average energy ejected electrons $\varepsilon_{\text {exp }}$ was obtained of the MODDEMAN [2], taking the average of the experimental values of each line of the spectral bands $\mathrm{B}, \mathrm{C}$ and D. The energies $E_{k}$ are the binding energies of the $\mathrm{K}$ shell. The $\left(E_{G S}-E_{f}\right)$ energies are the difference between energy of ground state $E_{G S}$ of the system and the highest energy of the normal Auger line $E_{f}$ obtained by Equation (10) of the text. A brief analysis of the results on Table 1 shows that the normal Auger GS $\rightarrow$ K-WW affects the calculated values of average energies $\varepsilon_{f G S}$, which are very close to the experimental values. We see that the inelastic collision theory can be used to predict the average energies band of ejection electrons Auger normal.

\section{REFERENCES}

1. Auger, P. (1925) Sur l'effet photoélectrique composé. Journal de Physique et Le Radium, 6, 205-208. https://doi.org/10.1051/jphysrad:0192500606020500

2. Moddeman, W.E., et al. (1971) Determination of the K-LL Auger Spectra of $\mathrm{N}_{2}, \mathrm{O}_{2}, \mathrm{CO}, \mathrm{NO}, \mathrm{H}_{2} \mathrm{O}$ and $\mathrm{CO}_{2}$. The Journal of Chemical Physics, 5, 2317.

3. Dias, A.M. (1994) Estudo do Efeito Auger em Moléculas pela Teoria das Colisões Inelásticas. Rev da Univ. de Alfenas, 1, 51-54.

4. Shirley, D.A. (1973) Theory of KLL Auger Energies Including Static Relaxation. Physical Review A, 7, $1520-1528$. https://doi.org/10.1103/PhysRevA.7.1520

5. Siegbahn, H., Asplund, L. and Kelfve, P. (1980) The Auger Spectrum of Water Vapour. Chemical Physics Letters, 69, 435-440.

6. Jennison, D.R. (1981) Initial-State Relaxation Effects in Molecular Auger Spectra. Physical Review A, 23, 1215-1222. https://doi.org/10.1103/PhysRevA.23.1215

7. Kleiber, J.A., Jennison, K.R. and Rye, R.R. (1981) Analysis of the Auger Spectra of CO and $\mathrm{CO}_{2}$. Journal of Chemical Physics, 75, 650-662. https://doi.org/10.1063/1.442104

8. Casanova, R., et al. (2002) Characterization of Iron Phyllosilicate Catalysts by Means of KLL Auger Spectra of Oxygen. Revista Latinoamericana de Metalurgia y Materiales, 22, 78-81.

9. Landau, L. and Lifchitz, E. (1966) Mecanique Quantique, Moscou, Ed MIR, 718 p. 